\section{Dick ohne Bierbauch: Ist das die Idealfigur gegen Prostatakrebs?}

\author{
Übergewichtige Männer entwickeln seltener ein Prostatakarzinom. Die Extra- \\ kilos sollten sich jedoch nicht um die Taille sammeln.
}

O b und wie Übergewicht das Auftreten eines Prostatakarzinoms (PCa) beeinflusst, ist nicht abschließend geklärt. Einer jetzt veröffentlichten FallKontroll-Studie zufolge, spielt nicht nur der Body-Mass-Index (BMI), sondern auch die Körperfettverteilung eine wesentliche Rolle.

In der Studie waren 1.933 kanadische Männer mit neu diagnostiziertem PCa mit einer Zufallsstichprobe von gleichaltrigen gesunden Männern verglichen worden. Männer mit einem BMI zwischen 25,0 und 29,9 hatten ein geringeres Risiko für ein $\mathrm{PCa}$ (Odds Ratio, OR $=0,87)$, und zwar sowohl für niedriggradige (Gleason-Score $\leq 6, \mathrm{OR}=0,83$ ) als auch für hochgradige $(\mathrm{OR}=0,89)$. Bei einem BMI ab 30 war die inverse Beziehung sogar noch etwas deutlicher.

Eine zentrale Adipositas (Taillenumfang $\geq 102 \mathrm{~cm}$ oder Taillen-Hüft-Verhältnis $>1,0)$ zeigte zunächst keine Assoziation zum Erkrankungsrisiko. Das änderte sich, wenn der Einfluss des BMI eliminiert wurde: Ein Taillenumfang $\geq 102 \mathrm{~cm}$ war dann mit einer erhöhten Rate von $\mathrm{PCa}(\mathrm{OR}=1,23)$, besonders in fortgeschrittenen Stadien $(\mathrm{OR}=1,47)$, verknüpft. Die Korrelation ließ sich sowohl bei übergewichtigen als auch bei adipösen Männern nachweisen. Auch ein TaillenHüft-Verhältnis > 1,0 ging bei konstantem BMI mit einer allerdings nur leicht erhöhten Wahrscheinlichkeit ausschließlich für hochgradige $\mathrm{PCa}$ einher $(\mathrm{OR}=1,20)$.
Fazit: „Unsere Daten sprechen dafür, dass die Körperfettverteilung bei einem gegebenen BMI ein Prädiktor für das Prostatakrebsrisiko ist", konstatieren die Autoren der Studie. BMI und zentrale Adipositas müssen daher gemeinsam betrachtet werden. Offenbar unterscheiden sich aber auch der Taillenumfang und das Taillen-Hüft-Verhältnis in ihrer Aussagekraft. „Der Taillenumfang ist ein Indikator für die viszerale Fettmasse und, besonders bei Männern, eng korreliert mit dem Anteil der Fettmasse", schreiben die Autoren. Er erfasse daher möglicherweise besser als das Taillen-Hüft-Verhältnis die für das Prostatakrebsrisiko relevante Verteilung von überzähligen Kilos.

Dr. Beate Schumacher

Boehm K et al. Waist circumference, waist-hip ratio, body mass index, and prostate cancer risk: Results from the North-American case-control study Prostate Cancer \& Environment Study. Urol Oncol 2015; doi: 10.1016/j.urolonc.2015.07.006.

\section{Tumorvolumen im Fokus: Kriterium für insignifikantes Prostatakarzinom im Wandel}

\author{
Beim Prostatakarzinom könnte eine Änderung der Kriterien für ein \\ insignifikantes Tumorleiden die Gefahr der Übertherapie reduzieren.
}

steherdrüse entfernt worden war, konnten australische Ärzte um Dr. Francis Ting jedoch keine Erhöhung der biochemischen Rezidivrate feststellen, wenn das Tumorvolumen statt unter $0,5 \mathrm{ml}$ zwischen 0,5 und 2,5 ml lag. Definiert war ein biochemisches Rezidiv als ein PSA-Wert $\geq 0,1 \mathrm{ng} / \mathrm{ml}$, was bei keinem Patienten im Follow-up-Zeitraum gemessen wurde.

Die Befunde von insgesamt 175 Patienten konnten für die Studie ausgewertet werden. Bei allen Männern war der Tumor zum Zeitpunkt der Prostatektomie auf das Organ beschränkt, die Schnittränder waren negativ. Der Gleason-Score lag bei 6 und das Tumorvolumen betrug weniger als 2,5 ml. Bei mehr als $78 \%$ der Patienten handelte es sich um Niedrigrisikotumoren, beurteilt anhand der
D'Amico-Kriterien PSA-Wert und Gleason-Score. Bei knapp 54 \% der Studienteilnehmer lag das Gesamtvolumen des Tumors unter $0,5 \mathrm{ml}$, bei den übrigen zwischen 0,5 und 2,5 $\mathrm{ml}$. Im Median betrug das Follow-up 74, höchstens 102 Monate.

Fazit: Ihr Studienergebnis sei ein weiteres Argument dafür, kleine Gleason-6-Karzinome als insignifikante Tumoren einzustufen. Einschränkend weisen die Ärzte darauf hin, dass die Zahl der Patienten geringer und das Follow-up kürzer als in anderen Studien gewesen sei. Allerdings gebe es Hinweise dafür, dass es ungefähr sieben Jahre dauert, bis sich ein niedriggradiges Karzinom in einen klinisch bedeutenden hochgradigen Tumor verwandelt. Deshalb gehen sie davon aus, dass in ihrer Studie die meisten potenziellen Prostatakarzinomrezidive erfasst worden sind, da mehr als 50 \% der Männer mindestens sieben Jahre lang nachbeobachtet werden konnten.

Peter Leiner

Ting F et al. Tumor volume in insignificant prostate cancer: increasing the threshold is a safe approach to reduce over-treatment. Prostate. 2015; doi: 10.1002/pros.23062. 